\title{
Nonalcoholic fatty liver disease: scintigraphy in the diagnosis of steatohepatitis
}

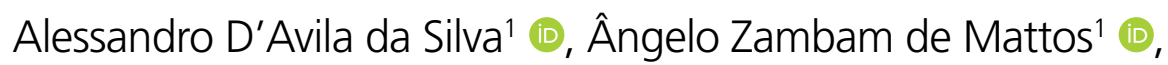 \\ Cristiane Valle Tovo ${ }^{*}$ (D), Larissa Faraco Daros² (D), Osvaldo Estrela Anselmi² (D), \\ Gabriela Perdomo Coral' ${ }^{1}$, Angelo Alves de Mattos $^{1}$
}

\section{SUMMARY}

OBJECTIVE: Nonalcoholic fatty liver disease is the most prevalent cause of chronic liver disease worldwide. Nonalcoholic steatohepatitis is associated with increased mortality rates due to the liver and cardiovascular diseases. The gold standard for discriminating nonalcoholic fatty liver disease activity and staging is the anatomopathological examination, which is an invasive method. In this regard, noninvasive methods, such as scintigraphy, have been under investigation. This study investigated the role of scintigraphy in the diagnosis of nonalcoholic steatohepatitis in obese patients with nonalcoholic fatty liver disease undergoing bariatric surgery.

METHODS: Patients undergoing bariatric surgery and liver biopsy were prospectively included. ${ }^{99 m}$ Tc-phytate scintigraphy was performed to assess liver/spleen, spleen/heart, and liver/heart uptake ratios, while ${ }^{99 \mathrm{~m} T c-i s o n i t r i l e ~ s c i n t i g r a p h y ~ a s s e s s e d ~ l i v e r / h e a r t ~ r a t i o . ~ T o ~ e v a l u a t e ~}$ the presence of nonalcoholic steatohepatitis, the results of ${ }^{99 m} \mathrm{Tc}$-phytate scintigraphy and ${ }^{99 \mathrm{~m} T c-i s o n i t r i l e ~ s c i n t i g r a p h y ~ w e r e ~ c o m p a r e d ~}$ with the anatomopathological examination.

RESULTS: Sixty-one patients with nonalcoholic fatty liver disease were allocated into two groups, namely, nonalcoholic steatohepatitis $(n=49)$ and non-nonalcoholic steatohepatitis $(n=12)$. The results of scintigraphic images obtained after the infusion of radiopharmaceutical ${ }^{99 m} \mathrm{mc}$-phytate in liver/spleen, spleen/heart, liver/heart ratios and ${ }^{99 \mathrm{~m}} \mathrm{Tc}$-isonitrile liver/heart ratio presented no difference between groups with and without nonalcoholic steatohepatitis with an accuracy of $47.5,37.7,50.8$, and $52.5 \%$, respectively.

CONCLUSION: Scintigraphy was not proven to be a useful method to differentiate patients with and without nonalcoholic steatohepatitis. KEYWORDS: Non-alcoholic fatty liver disease. Nonalcoholic steatohepatitis. Diagnosis. Radionuclide imaging.

\section{INTRODUCTION}

Nonalcoholic fatty liver disease (NAFLD) is the most frequent cause of chronic liver disease worldwide ${ }^{1,2}$. The clinical presentation of NAFLD ranges from simple steatosis to nonalcoholic steatohepatitis (NASH). NASH may progress to fibrosis, cirrhosis, and ultimately to hepatocellular carcinoma $(\mathrm{HCC})^{3}$. Furthermore, NAFLD is currently considered the hepatic manifestation of metabolic syndrome (MetS), which has well-documented associations with cardiovascular diseases, type 2 diabetes, and chronic kidney disease ${ }^{4}$. Patients with NASH present a higher mortality risk due to both liver and cardiovascular causes 5 .

Histological examination of the liver is the gold standard method for discriminating between the forms of NAFLD and for the diagnosis and staging of NASH. However, this is an invasive method. Additionally, due to the high prevalence rates of NAFLD, it is not suitable to perform a liver biopsy in most of the patients ${ }^{6,7}$.

\footnotetext{
'Universidade Federal de Ciências da Saúde de Porto Alegre, Hepatology Graduation Program - Porto Alegre (RS), Brazil.

${ }^{2}$ Santa Casa de Misericórdia de Porto Alegre, Nuclear Medicine Unit - Porto Alegre (RS), Brazil.

*Corresponding author: cristianev@ufcspa.edu.br

Conflicts of interest: the authors declare there are no conflicts of interest. Funding: none.

Received on July 29, 2021. Accepted on August 14, 2021.
} 
The available usual noninvasive methods do not allow discrimination between steatosis and steatohepatitis ${ }^{8-12}$, and since the currently existing study on the use of scintigraphy to assess $\mathrm{NASH}$ is scarce ${ }^{13,14}$, we aimed to evaluate the role of this method in the detection of inflammation in patients with NAFLD.

\section{METHODS}

Patients with clinical indications for bariatric surgery attended in Irmandade Santa Casa de Porto Alegre Hospital, a tertiary level hospital in southern Brazil, were prospectively included from October 2016 to March 2019.

Patients who underwent bariatric surgery according to formal indications ${ }^{15}$ were included.

All included patients underwent liver biopsy during surgery.

Patients with positive serological markers for hepatitis B virus and hepatitis $C$ virus, patients with significant alcohol consumption ( $>30 \mathrm{~g} /$ day for men and $20 \mathrm{~g} /$ day for women), and patients with chronic liver disease of other etiologies were not eligible for this study.

Information on gender, age, weight, height, body mass index (BMI), and the presence of comorbidities was recorded. The diagnosis of diabetes mellitus (DM) ${ }^{16}$, systemic arterial hypertension $(\mathrm{SAH}){ }^{17}$, and dyslipidemia ${ }^{18}$ followed the international recommendations. Additionally, the platelet count and biochemical parameters were evaluated. All laboratory tests were performed according to institutional standards and manufacturers' recommendations.

Histological analyses were performed by a pathologist blinded to clinical data. For the NASH diagnosis, the presence of steatosis associated with ballooning and the presence of inflammatory infiltrate was considered ${ }^{19,20}$.

Liver scintigraphy was performed on SIEMENS equipment (i.e., model Symbia T2, SPECT/CT technology, and 2-channel tomography), and the images were obtained using the Somaris/5 software, version 8.5.10.30.

After an 8-h fasting period and $20 \mathrm{~min}$ after the intravenous administration of $8 \mathrm{mCi}$ of Technetium-99m-phytate ( ${ }^{99 \mathrm{~m}} \mathrm{Tc}$-phytate), the patients underwent planar static image acquisition, including liver, spleen, and the cardiac area in the field of view, until 2100 million counts were reached, in a gamma $^{\text {Ts }} 2$-detector camera equipped with a low-energy, high-resolution collimator with parallel holes, $512 \times 512$ matrix, zoom 1.23. Regions of interest (ROIs) were designed in the liver, spleen, and heart, obtaining the average number of counts in these organs to calculate the liver/spleen, spleen/heart, and liver/heart ratios, according to the institutional protocol.

In the protocol with Technetium-99m-methoxy-isobutyl isonitrile $\left.{ }^{9{ }^{9} \mathrm{~m}} \mathrm{Tc}-\mathrm{MIBI}\right)$, after fasting for $8 \mathrm{~h}$ and $10 \mathrm{~min}$ after the intravenous administration of $16 \mathrm{mCi}$ of the radiopharmaceutical, patients underwent planar static image acquisition including the liver and the heart in the field of vision for 5 min, until reaching 1500 million counts in gamma-chamber. ROIs were drawn with the liver, right lobe, and heart in the field of view, obtaining the number of counts in these organs, to calculate the liver/heart ratio, following the institutional protocols. Liver scintigraphy was performed up to 1 month before surgery, and both protocols were performed on the same day.

In the statistical analyses, quantitative data were described as mean \pm standard deviation (SD). Median and minimum-maximum intervals were presented if the variables did not show normal distribution. Categorical data were presented by counts and percentages. Quantitative data were compared using the Mann-Whitney $\mathrm{U}$ test and categorical data using the Fisher's exact test. Diagnostic performance measures, such as sensitivity, specificity, and predictive values, were obtained. Statistical significance was set at $\mathrm{p}<0.05$.

\section{RESULTS}

Sixty-one patients were eligible for this study. Following the histopathological assessment of liver biopsies, patients with NAFLD were divided into two groups as follows: with NASH $(\mathrm{n}=49)$ and without NASH (steatosis only) $(\mathrm{n}=12)$.

Clinical and demographic characteristics were compared between groups (Table 1). The NASH group showed higher mean alanine aminotransferase (ALT) levels in comparison with the group without NASH. Regarding MetS, patients with NASH presented greater insulin resistance (IR). Although triglyceride levels were higher in patients with NASH, there was no difference in the frequency of dyslipidemia between groups. Likewise, despite the differences observed in the mean blood glucose values between groups, there was no difference in the distribution of DM between the groups with and without NASH.

The scintigraphic images obtained after infusion of the radiopharmaceutical ${ }^{99 \mathrm{~m}} \mathrm{Tc}$-phytate to assess the liver/spleen, spleen/heart, liver/heart ratios and $9^{9 \mathrm{~m}} \mathrm{Tc}-\mathrm{MIBI}$ for the liver/heart ratio were not different between groups (Table 2). After assessing sensitivity, specificity, positive predictive value (PPV), negative predictive value (NPV), and accuracy, the results did not reach the desired levels to validate scintigraphy as a method for the detection of NASH (Table 3).

\section{DISCUSSION}

Since NAFLD is the most common cause of liver disease and bearing in mind the different natural histories of patients with $\mathrm{NASH}$ and those with steatosis, a differential diagnosis is of the highest importance. 
Table 1. Distribution of demographic, clinical, and laboratory data of groups with and without steatohepatitis.

\begin{tabular}{|c|c|c|c|c|}
\hline & All-cases $(n=61)$ & Without NASH $(n=12)$ & With NASH $(n=49)$ & p-value \\
\hline Age* (years) & $39.4 \pm 9.1$ & $36.1 \pm 8.4$ & $40.2 \pm 9.2$ & $0.159^{a}$ \\
\hline Female** & $49(80.3)$ & $9(7.5)$ & $40(81.6)$ & $0.689^{a}$ \\
\hline $\mathrm{BMI}^{*}\left(\mathrm{~kg} / \mathrm{m}^{2}\right)$ & $42.5 \pm 5.5$ & $44.5 \pm 3.0$ & $42.1 \pm 5.7$ & $0.050^{a}$ \\
\hline$A L T *(U / L)$ & $36.3 \pm 21.2$ & $24.6 \pm 10.3$ & $39.1 \pm 22.0$ & $0.009^{a}$ \\
\hline $\mathrm{AST}^{*}(\mathrm{U} / \mathrm{L})$ & $26.4 \pm 11.1$ & $22.3 \pm 6.3$ & $27.4 \pm 1.8$ & $0.167^{a}$ \\
\hline Bilirubin* (mg/dL) & $0.4 \pm 0.3$ & $0.4 \pm 0.3$ & $0.4 \pm 0.3$ & $0.956^{a}$ \\
\hline Triglycerides* (mg/dL) & $177.3 \pm 160.8$ & $105.6 \pm 34.8$ & $198.9 \pm 74.5$ & $0.011^{a}$ \\
\hline $\mathrm{TC} *(\mathrm{mg} / \mathrm{dL})$ & $193.9 \pm 38.6$ & $174.8 \pm 30.4$ & $198.5 \pm 39.3$ & $0.059^{a}$ \\
\hline $\mathrm{HDL}^{*}(\mathrm{mg} / \mathrm{dL})$ & $47.1 \pm 10.4$ & $53.6 \pm 11.2$ & $45.6 \pm 9.7$ & $0.038^{a}$ \\
\hline $\mathrm{LDL}^{*}(\mathrm{mg} / \mathrm{dL})$ & $114 \pm 29$ & $100 \pm 26$ & $117 \pm 28$ & $0.106^{a}$ \\
\hline Glucose* (mg/dL) & $104.5 \pm 33.0$ & $87.2 \pm 8.8$ & $108.8 \pm 35.3$ & $0.004^{a}$ \\
\hline Insulin* (U/L) & $20.7 \pm 9.1$ & $16.8 \pm 5.8$ & $21.7 \pm 9.5$ & $0.037^{a}$ \\
\hline HOMA-IR* & $5.5 \pm 3.3$ & $3.8 \pm 1.5$ & $6.0 \pm 3.5$ & $0.013^{a}$ \\
\hline Platelets* $\left(\mathrm{mm}^{3}\right)$ & $274573 \pm 67274$ & $272000 \pm 68747$ & $275204 \pm 67616$ & $0.949^{a}$ \\
\hline Ferritin* $(\mathrm{ng} / \mathrm{mL})$ & $240.9 \pm 226.6$ & $214.6 \pm 157.2$ & $247.3 \pm 241.5$ & $0.986^{a}$ \\
\hline Albumin* (g/dL) & $4.2 \pm 0.3$ & $4.2 \pm 0.3$ & $4.2 \pm 0.3$ & $0.964^{a}$ \\
\hline Hypertension ** & $28(45.9)$ & $3(25.0)$ & $25(51.0)$ & $0.122^{b}$ \\
\hline Diabetes** & $14(23.0)$ & $1(8.3)$ & $13(26.5)$ & $0.264^{b}$ \\
\hline Dyslipidemia** & $12(19.7)$ & $0(0)$ & $12(24.5)$ & $0.100^{\mathrm{b}}$ \\
\hline
\end{tabular}

NASH: nonalcoholic steatohepatitis; BMI: body mass index; HOMA-IR: homeostasis model assessment-estimated insulin resistance; ALT: alanine aminotransferase; AST: aspartate aminotransferase; TC: total cholesterol. *Data presented as mean \pm SD; **Data presented as number of patients (\% of all patients). ${ }^{\mathrm{M} M a n n-W h i t n e y ~} \mathrm{U}$ test. ${ }^{\circ}$ Fisher's exact test.

Table 2. Comparison of scintigraphic ratios between patients with and without nonalcoholic steatohepatitis.

\section{All-cases $(n=61) \quad$ Without NASH $(n=12) \quad$ With NASH $(n=49) \quad p$-value}

99mTc-phytate liver/spleen

\begin{tabular}{|c|c|c|c|c|}
\hline Mean $\pm S D$ & $5.73 \pm 2.60$ & $7.43 \pm 4.46$ & $5.32 \pm 1.73$ & \multirow{3}{*}{0.095} \\
\hline Median & 5.35 & 7.05 & 5.31 & \\
\hline$(\min -\max )$ & $2.67-19.49$ & $3.8-19.49$ & $2.67-10.59$ & \\
\hline \multicolumn{5}{|c|}{${ }^{99 \mathrm{~m} T \mathrm{Tc}-\text { phytate spleen/heart }}$} \\
\hline Mean \pm SD & $2.25 \pm 0.65$ & $2.03 \pm 0.62$ & $2.30 \pm 0.65$ & \multirow{3}{*}{0.207} \\
\hline Median & 2.17 & 1.99 & 2.26 & \\
\hline$(\min -\max )$ & $1.01-4.23$ & $1.01-3.33$ & $1.30-4.23$ & \\
\hline \multicolumn{5}{|c|}{${ }^{99 \mathrm{~m}} \mathrm{Tc}$-phytate liver/heart } \\
\hline Mean $\pm S D$ & $11.90 \pm 3.08$ & $13.21 \pm 3.89$ & $11.58 \pm 2.80$ & \multirow{3}{*}{0.179} \\
\hline Median & 11.85 & 12.84 & 11.56 & \\
\hline (min-max) & $6.10-19.84$ & $6.81-19.84$ & $6.10-17.96$ & \\
\hline \multicolumn{5}{|c|}{ 99m TC-MIBIliver/heart } \\
\hline Mean $\pm S D$ & $2.08 \pm 0.71$ & $2.32 \pm 0.84$ & $2.02 \pm 0.67$ & \multirow{3}{*}{0.171} \\
\hline Median & 1.92 & 2.19 & 1.92 & \\
\hline (min-max) & $1.11-4.31$ & $1.51-4.31$ & $1.11-4.26$ & \\
\hline
\end{tabular}

NASH: nonalcoholic steatohepatitis; MIBI: methoxy-isobutyl isonitrile. ${ }^{\circledR}$ Mann-Whitney $\mathrm{U}$ test. 
Table 3. Sensitivity, specificity, positive predictive value, negative predictive value, and accuracy of scintigraphy in the diagnosis of nonalcoholic steatohepatitis.

\begin{tabular}{|c|c|c|c|c|c|}
\hline & Sensitivity (\%) & Specificity (\%) & PVV (\%) & NPV (\%) & Accuracy (\%) \\
\hline${ }^{99 \mathrm{~m} T \mathrm{Tc}-\text { phytateliver/spleen } \leq 5}$ & 44.9 & 58.3 & 81.5 & 20.6 & 47.5 \\
\hline 99mTc-phytatespleen/heart $\leq 2$ & 34.7 & 50.0 & 73.9 & 15.8 & 37.7 \\
\hline${ }^{99 \mathrm{~m} T c-p h y t a t e l i v e r / h e a r t \leq 11.5}$ & 46.9 & 66.7 & 85.2 & 23.5 & 50.8 \\
\hline $\begin{array}{l}\text { 99mTc-methoxy-isobutyl } \\
\text { isonitrileliver/heart } \leq 1.9\end{array}$ & 49 & 66.7 & 85.7 & 24.2 & 52.5 \\
\hline
\end{tabular}

PVV: positive predictive value; NPV: negative predictive value.

The identification of inflammation by the assessment of serum biomarkers is expensive, poorly available, and presents a low accuracy. In turn, imaging methods are a recognized approach for the detection of only steatosis and fibrosis but are limited for the diagnosis of $\mathrm{NASH}^{12}$. Among them, scintigraphy may represent a low-risk and relatively low-cost method, thus favoring its employment for the identification of inflammation in patients with NAFLD. It has been demonstrated that the reticuloendothelial dysfunction of Kupffer cells contributes to the pathogenesis of steatohepatitis. As ${ }^{99 \mathrm{~m}} \mathrm{Tc}$-phytate accumulates in the liver followed by phagocytosis by Kupffer cells, the reduction of this radioisotope uptake of during the scintigraphic exam could be a sensitive marker of steatosis to NASH progression ${ }^{13}$. Similarly, due to the hepatic mitochondrial abnormalities described in the pathogenesis of NASH, the decreased uptake of ${ }^{99 \mathrm{~m}} \mathrm{Tc}$-MIBI could also reveal clinical significance, as it indicates progression to a more advanced disease stage $e^{14}$.

To the best of our knowledge, only two studies have compared the role of scintigraphy between patients with NASH and simple steatosis to the present time ${ }^{13,14}$. Kikuchi et al. ${ }^{13}$ evaluated 37 patients with suspected NAFLD at the time of liver biopsy ( 8 patients with simple steatosis and 29 with NASH). A reduced ${ }^{99 \mathrm{~m}}$ Tc-phytate liver-spleen ratio was demonstrated in the NASH group in relation to the group with simple steatosis. Importantly, the decrease in the liver/spleen ratio was observed in all NASH stages, and it was identified as an independent predictor to distinguish NASH and steatosis. Masuda et al. ${ }^{14}$ employed the radiopharmaceutical ${ }^{99 \mathrm{~m}} \mathrm{Tc}$-MIBI to evaluate 26 patients with biopsy-proven NAFLD (four with simple steatosis, 11 with borderline NASH, and 11 with $\mathrm{NASH}$ ). The authors reported a significantly lower liver-heart ratio in the NASH group compared with the steatosis group. In contrast to these results, which support the use of scintigraphy in the diagnosis of NASH, we did not identify a difference in ${ }^{99 \mathrm{~m}} \mathrm{Tc}$-phytate or $9^{9 \mathrm{~m}} \mathrm{Tc}$-MIBI uptake between the groups with and without NASH. Unlike the above-mentioned studies, despite having assessed the ROIs in order to include the liver tissue to a greater extent, our results did not provide the expected superior accuracy.

Furthermore, in contrast to the population included in this study, whose mean BMI was $42.5 \mathrm{~kg} / \mathrm{m}^{2}$, the patients included in the studies of Kikuchi et al. ${ }^{13}$ and Masuda et al. ${ }^{14}$ presented an average BMI of $25-30 \mathrm{~kg} / \mathrm{m}^{2}$. In fact, the patients included in this study were obese who underwent bariatric surgery, which could possibly have a higher degree of inflammation, thus favoring a more precise distinction between groups.

Despite this study having been prospectively performed and all included patients having undergone histological evaluation, the main limitation was the small sample size, particularly in the group of patients without NASH. However, this study included 61 patients, while the studies by Kikuchi et al. ${ }^{13}$ and Masuda et al. ${ }^{14}$ have evaluated 37 and 26 patients, respectively.

\section{CONCLUSION}

We believe that it was not possible to demonstrate the usefulness of scintigraphy to discriminate groups of patients with or without NASH.

\section{AUTHORS" CONTRIBUTION}

ADS: Conceptualization, Data curation, Formal analysis, Writing - original draft, Writing - review \& editing. AAM: Conceptualization, Data curation, Formal analysis, Writing original draft, Writing - review \& editing. CVT: Data curation, Formal analysis, Writing - original draft, Writing - review \& editing. LFD: Data curation, Formal analysis, Writing - original draft, Writing - review \& editing. OEA: Data curation, Formal analysis, Writing - original draft, Writing - review \& editing. GPC: Data curation, Formal analysis, Writing - original draft, Writing - review \& editing. AAM: Data curation, Formal analysis, Writing - original draft, Writing - review \& editing 


\section{REFERENCES}

1. Younossi ZM, Koenig $A B$, Abdelatif $D$, Fazel $Y$, Henry $L$, Wymer M. Global epidemiology of nonalcoholic fatty liver disease-Meta-analytic assessment of prevalence, incidence, and outcomes. Hepatology. 2016;64(1):73-84. https://doi. org/10.1002/hep.28431

2. Doycheva I, Watt KD, Alkhouri N. Nonalcoholic fatty liver disease in adolescents and young adults: The next frontier in the epidemic. Hepatology. 2017;65(6):2100-9. https://doi. org/10.1002/hep.29068

3. Chalasani N, Younossi Z, Lavine JE, Diehl AM, Brunt EM, Cusi K, et al. The diagnosis and management of non-alcoholic fatty liver disease: practice Guideline by the American Association for the Study of Liver Diseases, American College of Gastroenterology, and the American Gastroenterological Association. Hepatology. 2012;55(6):2005-23. https://doi.org/10.1002/hep.25762

4. Boppidi H, Daram SR. Nonalcoholic fatty liver disease: hepatic manifestation of obesity and the metabolic syndrome. Postgrad Med. 2008;120(2):E01-7. https://doi.org/10.3810/ pgm.2008.07.1800

5. Younossi ZM, Stepanova M, Rafiq N, Henry L, Loomba R, Makhlouf H, Goodman Z. Nonalcoholic steatofibrosis independently predicts mortality in nonalcoholic fatty liver disease. Hepatol Commun. 2017;1(5):421-8. https://doi. org/10.1002/hep4.1054

6. Bedossa P, Patel K. Biopsy and noninvasive methods to assess progression of nonalcoholic fatty liver disease. Gastroenterology. 2016;150(8):1811-22.e4. https://doi. org/10.1053/j.gastro.2016.03.008

7. Angulo P, Kleiner DE, Dam-Larsen S, Adams LA, Bjornsson ES, Charatcharoenwitthaya $P$, et al. Liver fibrosis, but no other histologic features, is associated with long-term outcomes of patients with non-alcoholic fatty liver disease. Gastroenterology. 2015;149(2):389-97.e10. https://doi. org/10.1053/j.gastro.2015.04.043

8. Kruger FC, Daniels CR, Kidd M, Swart G. Brundyn K, Van Rensburg C, et al. APRI: a simple bedside marker for advanced fibrosis that can avoid liver biopsy in patients with NAFLD/ NASH. S Afr Med J. 2011;101(7):477-80. PMID: 21920102

9. Shah AG, Lydecker A, Murray K, Tetri BN, Contos MJ, Sanyal $A J$, et al. Use of the FIB4 index non-invasive evaluation of fibrosis in non-alcoholic fatty liver disease. Clin Gastroenterol Hepatol. 2009;7(10):1104-12. https://doi.org/10.1016/j. cgh.2009.05.033

10. Angulo P, Hui JM, Marchesini G, Bugianesi E, George J, Farrell $G C$, et al. The NAFLD fibrosis score: a non-invasive system that identifies liver fibrosis in patients with NAFLD. Hepatology. 2007;45(4):846-54. https://doi.org/10.1002/hep.21496

11. Morra R, Munteanu M, Imbert-Bismut F, Messous D, Ratziu $V$, Poynard T. FibroMAX: towards a new universal biomarker of liver disease? Expert Rev Mol Diagn. 2007;7(5):481-90. https://doi.org/10.1586/14737159.7.5.481

12. Tovo CV, Mattos AZ, Coral GP, Branco FS, Suwa E, Mattos AA. Noninvasive imaging assessment of non-alcoholic fatty liver disease: focus on liver scintigraphy. World J Gastroenterol. 2015;21(15):4432-9. https://doi.org/10.3748/wjg.v21.i15.4432

13. Kikuchi M, Tomita K, Nakahara T, Kitamura N, Teratani T, Irie

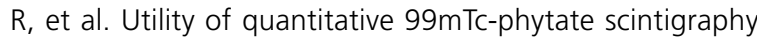
to diagnose early-stage non-alcoholic steatohepatitis. Scand J Gastroenterol. 2009;44(2):229-36. https://doi. org/10.1080/00365520802433249

14. Masuda K, Ono M, Fukumoto M, Hirose A, Munekage K, Ochi T, et al. Usefulness of Technetium-99 m-2-methoxy-isobutylisonitrile liver scintigraphy for evaluating disease activity of non-alcoholic fatty liver disease. Hepatol Res. 2012;42(3):273-9. https://doi.org/10.1111/j.1872-034X.2011.00923.x

15. Garvey WT, Mechanick JI, Brett EM, Garber AJ, Hurley $\mathrm{DL}$, Jastreboff AM et al. American association of clinical endocrinologists and american college of endocrinology comprehensive clinical practice guidelines for medical care of patients with obesity. Endocr Pract. 2016;22(Suppl 3):1-203. https://doi.org/10.4158/EP161365.GL

16. American Diabetes Association. Classification and diagnosis of diabetes: standards of medical care in diabetes-2018. Diabetes Care. 2018;41(Suppl 1):S13-27. https://doi.org/10.2337/ dc18-S002

17. Whelton PK, Carey RM, Aronow WS, Casey Junior DE, Collins KJ, Dennison-Himmelfarb C, et al. 2017 ACC/AHA/AAPA ABC/ACPM/AGS/APhA/ASH/ ASPC/NMA/PCNA Guideline for the prevention, detection, evaluation, and management of high blood pressure in adults: executive summary: a report of the American College of Cardiology/American Heart Association Task Force on Clinical Practice Guidelines. Hypertension. 2018;71(6):1269-324. https://doi.org/10.1161/ HYP.0000000000000066

18. Grundy SM, Stone NJ, Bailey AL, Beam C, Birtcher KK, Blumenthal RS, et al. 2018 Guideline on the Management of Blood Cholesterol: executive summary: a report of the American College of Cardiology/American Heart Association Task Force on Clinical Practice Guidelines. J Am Coll Cardiol. 2019;73(24):3168-209. https://doi.org/10.1016/j. jacc.2018.11.002

19. Kleiner DE, Brunt ME, Van Natta M, Behling C, Contos MJ, Cumming OW, et al. Design and validation of a histological scoring system for non-alcoholic fatty liver disease. Hepatology. 2005;41(6):1313-21. https://doi.org/10.1002/hep.20701

20. Kleiner DE, Brunt EM. Non-alcoholic fatty liver disease: pathologic patterns and biopsy evaluation in clinical research. Semin Liver Dis. 2012;32(1):3-13. https://doi.org/10.1055/s-0032-1306421 